\title{
Simple vibration modeling of structural fuzzy with continuous boundary by including two-dimensional spatial memory
}

Friis, Lars; Ohlrich, Mogens

Published in:

Journal of the Acoustical Society of America

Link to article, DOI:

$10.1121 / 1.2932077$

Publication date:

2008

Document Version

Publisher's PDF, also known as Version of record

Link back to DTU Orbit

Citation (APA):

Friis, L., \& Ohlrich, M. (2008). Simple vibration modeling of structural fuzzy with continuous boundary by including two-dimensional spatial memory. Journal of the Acoustical Society of America, 124(1), 192-202. https://doi.org/10.1121/1.2932077

\section{General rights}

Copyright and moral rights for the publications made accessible in the public portal are retained by the authors and/or other copyright owners and it is a condition of accessing publications that users recognise and abide by the legal requirements associated with these rights.

- Users may download and print one copy of any publication from the public portal for the purpose of private study or research.

- You may not further distribute the material or use it for any profit-making activity or commercial gain

- You may freely distribute the URL identifying the publication in the public portal 


\title{
Simple vibration modeling of structural fuzzy with continuous boundary by including two-dimensional spatial memory
}

\author{
Lars Friis ${ }^{a)}$ \\ Acoustic Technology, Ørsted·DTU, Technical University of Denmark, Building 352, DK-2800 Kgs. Lyngby, \\ Denmark and Widex A/S, Ny Vestergaardsvej 25, DK-3500 Vaerloese, Denmark \\ Mogens Ohlrich ${ }^{\text {b) }}$ \\ Acoustic Technology, Ørsted·DTU, Technical University of Denmark, Building 352, DK-2800 Kgs. Lyngby, \\ Denmark
}

(Received 6 November 2007; revised 8 April 2008; accepted 28 April 2008)

\begin{abstract}
Many complicated systems of practical interest consist basically of a well-defined outer shell-like master structure and a complicated internal structure with uncertain dynamic properties. Using the "fuzzy structure theory" for predicting audible frequency vibration, the internal structure is considered as one or more fuzzy substructures that are known in some statistical sense only. Experiments have shown that such fuzzy substructures often introduce a damping in the master which is much higher than the structural losses account for. A special method for modeling fuzzy substructures with a one-dimensional continuous boundary was examined in a companion paper [L. Friis and M. Ohlrich, "Vibration modeling of structural fuzzy with continuous boundary," J. Acoust. Soc. Am. 123, 718-728 (2008)]. In the present paper, this method is extended, such that it allows modeling of fuzzy substructures with a two-dimensional continuous boundary. Additionally, a simple method for determining the so-called equivalent coupling factor is presented. The validity of this method is demonstrated by numerical simulations of the vibration response of a master plate structure with fuzzy attachments. It is revealed that the method performs very well above a nondimensional frequency of 500 of the master, and it is shown that errors below this frequency are caused mainly by simplifying assumptions concerning the shape of the master vibration displacement. (C) 2008 Acoustical Society of America. [DOI: 10.1121/1.2932077]
\end{abstract}

PACS number(s): 43.40.At, 43.40.Tm [DF] Pages: 192-202

\section{INTRODUCTION}

For about 20 years, the "theory of fuzzy structures"1-3 has been known as a suggested alternative method for predicting the vibration of complex systems having many degrees of freedom and uncertain properties. By using this theory, a system is divided into a well-defined master structure and one or more complex parts termed as fuzzy substructures. It is assumed that the deterministic master, which is typically a shell-like structure, can be modeled by using traditional methods, whereas the fuzzy has imprecisely known properties that are known only in some statistical sense. Examples of real-life fuzzy systems varying from small size to large size are electro-mechanical hearing aids, machines, aircraft, and ship hulls.

Experiments have shown that such fuzzy attachments seemingly introduce high damping in the master structure, due to the dissipation of energy into the many degrees of freedom. The theory of fuzzy structures explains this damping effect, caused by multiple reflections rather than actual damping, by regarding the dynamic behavior of the fuzzy similar to that of a multitude of dynamic neutralizers or absorbers. ${ }^{4-6}$ Despite of this relatively simple hypothesis, publications on experimental investigations and practical use

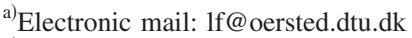

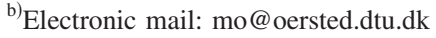

of the theory of fuzzy structures have so far been very limited in open literature. This is not only partly due to difficulties in determining the fuzzy parameters but also because of complicating issues such as the incorporation of different motion coordinates and modeling of fuzzy structures connected to the master through a continuous boundary.

The present paper examines and extends a special method of modeling structural fuzzy with a continuous boundary. This method was originally formulated by Soize and briefly presented in a paper from 1993. ${ }^{3}$ A successful modeling of structural fuzzy with continuous boundary requires that its stiffness must be taken into account. Soize achieved this by introducing spatial memory in the fuzzy boundary impedance. However, such boundary impedance is nonlocal and, therefore, laborious to implement in numerical methods. A full implementation is, therefore, circumvented by introducing an equivalent coupling factor that converts the distributed impedance to a local form. Soize's method of including spatial memory is clearly innovative; however, in the author's opinion, the main ideas of the method need clarification and physical interpretation.

In a companion paper, ${ }^{7}$ Soize's method was thoroughly examined and physical interpretations were offered. Furthermore, the smoothed fuzzy boundary impedance was formulated from simple mathematics without the use of probabilistic concepts. The present paper contributes with an extension of the method to two dimensions and with a simple and 
general method for determining the equivalent coupling factor. This method is examined through numerical simulations and its limitations are discussed. The companion paper and the present paper represent a continuation of the papers by Pierce et al. ${ }^{8}$ and Strasberg and Feit ${ }^{9}$ but for structural fuzzy with continuous boundary.

The theory of fuzzy structures was originally developed by Soize and presented in a series of papers ${ }^{1-3}$ from 1986 to 1993. During the last 20 years, the literature has partly focused on interpretation and simplification of Soize's theory that involves probabilistic concepts in order to account for model uncertainties. One milestone was the publications of simple and deterministic methods by Pierce et al. ${ }^{8}$ and Strasberg and Feit. ${ }^{9}$ These papers clarified the main concepts of the theory of fuzzy structures and have provided simple procedures for predicting the smoothed average response of complex systems. Furthermore, it was revealed that the damping induced in the master was governed mainly by the frequency-dependent resonating mass per unit frequency of the fuzzy. Several authors have examined the fuzzy damping effect in great detail. This includes Maidanik and Becker ${ }^{10-12}$ who unambiguously demonstrated the nature of the damping and set up design rules for complex attachments. The damping caused by different local oscillators was likewise investigated by Maidanik and Becker. ${ }^{13,14}$ Moreover, Weaver ${ }^{15}$ and Carcaterra and Akay ${ }^{16}$ revealed that the fuzzy damping is a transient phenomenon in the case of a finite number of complex attachments. It was shown that the energy returns to the master at later times when excited by a transient. One of the most difficult challenges in applying the theory of fuzzy structures is the determination of the resonating mass per unit frequency. During the last ten years, both Soize ${ }^{17,18}$ and Pierce ${ }^{19}$ addressed this problem. Another important highlight was the development of a method ${ }^{3}$ for including spatial memory in the modeling of structural fuzzy with continuous boundaries. With the exception of a few publications, ${ }^{7,17,18}$ succeeding literature has mainly focused on developing methods that regard the structural fuzzy as local fuzzy substructures. Many real-life structures, however, involve fuzzy structures with continuous boundaries, and the authors of the present paper believe that further study in this area is strongly needed in order to clarify some of the main ideas.

In favor of the reader, the method of including spatial memory in structural fuzzy will be briefly outlined in Sec. II below. Hereafter, a general method of determining the equivalent coupling factor is presented for a master structure with one-dimensional wave motion. Next, in Sec. IV, the fuzzy boundary impedance will be derived for structural fuzzy attached to the master through an area. After this, the method of finding the equivalent coupling factor is extended to two-dimensional wave motion in the master structure. Finally, in Sec. V, the method will be validated through numerical simulations and its usability and limitations will be discussed in Section VI.

\section{STRUCTURAL FUZZY WITH CONTINUOUS BOUNDARY}

\section{A. Introduction}

Fuzzy structure theory is intended for predicting the vibration and damping induced in a master structure due to one or more fuzzy substructures. The method is applicable mainly in the midfrequency range, where the master structure has well separated modes and where the fuzzy is highly resonant. Since the fuzzy is more or less compliantly attached to the master, the fuzzy behaves predominantly similar to a large number of "sprung masses" or "dynamic neutralizers" resonating at different frequencies. If these resonance frequencies of the fuzzy are closely spaced, then the fuzzy substructure will minimize and absorb vibration energy from the master over a considerable frequency band. Consequently, by considering the vibration response of the master, it appears as if the master is highly damped.

In the theory of fuzzy structures, each fuzzy substructure is modeled as infinitely many dynamic neutralizers attached to the connection surface. These neutralizers have different masses and their resonance frequencies are closely spaced, and altogether they, therefore, introduce a frequencydependent damping in the master. Further, it is assumed that the total mass of all the oscillators is equal to the mass of the fuzzy substructure that is to be modeled. A fuzzy substructure is typically separated from the master and conveniently modeled in terms of its boundary impedance $\underline{z}_{\text {fuzzy, } \varepsilon}\left(x_{0}, y_{0} ; x_{1}, y_{1}\right)$. This boundary impedance expresses the relationship between the force per unit area $F_{\prime \prime}\left(x_{0}, y_{0}\right)$ induced at $\left(x_{0}, y_{0}\right)$ due to the velocity $\underline{v}\left(x_{1}, y_{1}\right)$ of an infinitesimal area element $d A$ at $\left(x_{1}, y_{1}\right)$, while all other positions are locked, that is,

$$
\begin{aligned}
& \underline{F}^{\prime \prime}\left(x_{0}, y_{0}\right) \\
& \quad=\left.\underline{z}_{\text {fuzzy, }, \varepsilon}\left(x_{0}, y_{0} ; x_{1}, y_{1}\right) \underline{v}\left(x_{1}, y_{1}\right) d A\right|_{\underline{\underline{u}}\left[(x, y) \neq\left(x_{1}, y_{1}\right)\right]=0} .
\end{aligned}
$$

\section{B. The spatial oscillator}

In many cases, the fuzzy substructure is attached to the master through a continuous boundary. This also implies that the stiffness distribution of the fuzzy has to be taken into account and that the associated transfer terms of the impedance in Eq. (1) can only be neglected in special cases. ${ }^{7}$ Consider a fuzzy substructure connected to the master structure through a continuous boundary. Generally, this continuous boundary will be a surface, but for the sake of simplicity, we shall here consider a fuzzy attached to the master through a one-dimensional boundary of length $L_{\text {fuzzy }}$. Soize ${ }^{3}$ incorporates the stiffness of such a fuzzy substructure by including a spatial memory in the fuzzy boundary impedance. This is accomplished by introducing $a$ spatial oscillator as sketched in Fig. 1(a). The oscillator is defined by its stiffness width or spatial memory $2 \varepsilon$, the point mass $M$, the lossfactor $\eta$, and the resonance frequency $f_{r}$. Furthermore, it has a stiffness density distribution $\underline{s}_{\varepsilon}\left(x^{\prime}-x_{1}\right)$ that only depends on $\left(x^{\prime}-x_{1}\right)$ and which is defined as 

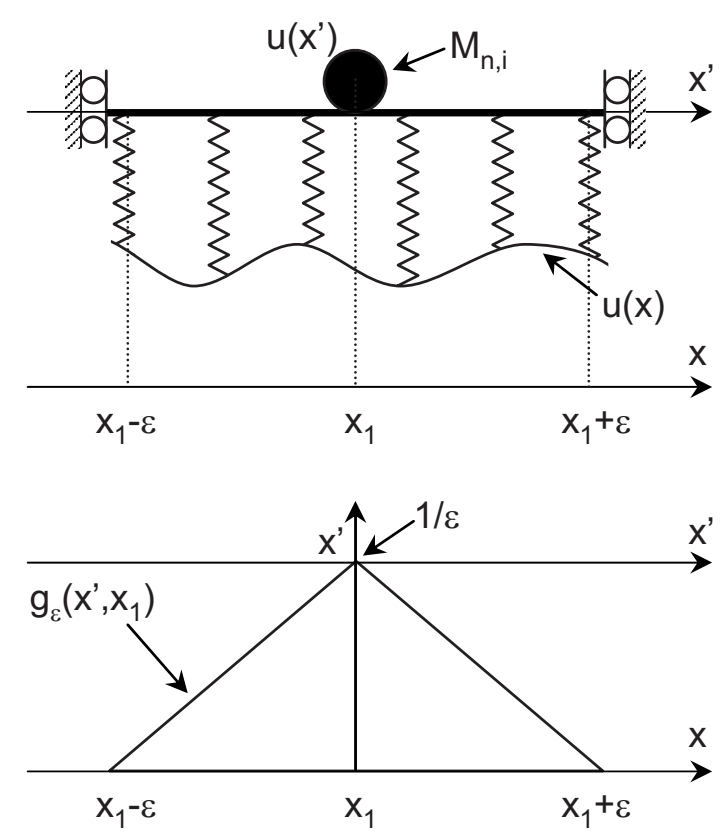

FIG. 1. Fuzzy oscillator with spatial memory. (a) Oscillator attached to a boundary of motion $u(x)$ and (b) stiffness distribution function of the oscillator springs $g_{\varepsilon}\left(x^{\prime}-x_{1}\right)$

$$
\underline{s}_{\varepsilon}\left(x^{\prime}-x_{1}\right)=\underline{s}_{\varepsilon}\left(x^{\prime}-x_{1}\right)=\left(M \omega_{r}^{2}\right)(1+i \eta) g_{\varepsilon}\left(x^{\prime}-x_{1}\right),
$$

where $\omega_{r}=2 \pi f_{r}$ is the angular resonance frequency and $\underline{s}$ is the complex total stiffness of the oscillator. The distribution function $g_{\varepsilon}\left(x^{\prime}-x_{1}\right)$ is an even function with an area of 1 . As a one-dimensional spatial memory, Soize suggests ${ }^{3}$ that $g_{\varepsilon}\left(x^{\prime}-x_{1}\right)$ is a triangular distribution, as shown in Fig. 1(b). This distribution is given as

$$
g_{\varepsilon}\left(x^{\prime}-x_{1}\right)=g_{\varepsilon}\left(x_{1}-x^{\prime}\right)=\frac{\varepsilon-\left|x^{\prime}-x_{1}\right|}{\varepsilon^{2}} 1_{\left[x_{1} \in\left(x^{\prime}-\varepsilon, x^{\prime}+\varepsilon\right)\right]},
$$

where $1_{\left[x_{1} \in\left(x^{\prime}-\varepsilon, x^{\prime}+\varepsilon\right)\right]}$ is a function, which is equal to 1 when $x_{1} \in\left[x^{\prime}-\varepsilon, x^{\prime}+\varepsilon\right]$ and 0 elsewhere. The spatial oscillator is discussed in more detail in Ref. 7.

\section{Sets of infinitely many identical oscillators}

Let us consider a fuzzy substructure with spatial memory connected to the master over a length $L_{\text {fuzzy }}$. Such a substructure comprises a double infinity of spatial oscillators, as sketched in Fig. 2, where each oscillator is depicted as a point mass and the triangular stiffness distribution shown in Fig. 1(b). First, the oscillators are grouped into sets of infinitely many identical oscillators overlapping one another such that each position on $L_{\text {fuzzy }}$ is associated with a point mass. Second, the structural fuzzy consists of infinitely many different sets, each with its individual resonance frequency, mass, and spatial memory.

Now, the $n$th set $(n \in[1, \infty[)$ of spatial oscillators is shown in Fig. 3(a). This set has a resonance frequency $f_{r, n}$, a total mass of $M_{n}$, and a spatial memory of $2 \varepsilon_{n}$. Further, the $i$ th spatial oscillator $(i \in[1, \infty[)$ of this $n$th set has the natural frequency $f_{r, n, i}=f_{r, n}$, a mass $M_{n, i}=M_{n} / L_{\text {fuzzy }}$, and a total stiff-

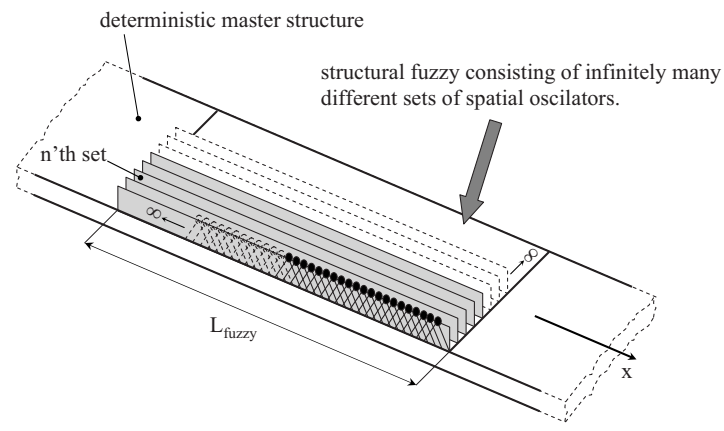

FIG. 2. Master structure undergoing one-dimensional wave motion with a fuzzy substructure attached through a one-dimensional continuous boundary in the $x$ direction. The fuzzy substructure has infinitely many sets of spatial oscillators and the $n$th set consists of infinitely many identical oscillators with resonance frequency $f_{r, n, i}$, mass $M_{n, i}$, and spatial memory $\varepsilon_{n}$.

ness $\underline{s}_{n, i}$. The relationship between the force per unit length $F_{\varepsilon, n}^{\prime}\left(x_{0}\right)$ at $x_{0}$ and the velocity $v\left(x_{1}\right)$ at $x_{1}$, that is, the boundary impedance of the $n$th set $z_{\varepsilon, n}\left(x_{0}-x_{1}, f_{r, n}\right)$, was derived in Ref. 1 . When multiplied by $d x_{1}$, this is given as

$$
\begin{aligned}
\underline{z}_{\varepsilon, n}\left(x_{0}-x_{1}, f_{r, n}\right) d x_{1}= & \frac{\underline{S}_{n, i}}{i \omega}\left[\delta_{x_{0}, x_{1}}-\frac{\underline{S}_{n, i}}{\underline{S}_{n, i}-\omega^{2} M_{n, i}}\left(g_{\varepsilon_{n}} * g_{\varepsilon_{n}}\right)\right. \\
& \left.\times\left(x_{0}-x_{1}\right) d x_{1}\right] \\
= & -i \omega\left(\frac{f_{r, n}^{2}}{f^{2}}\right)(1+i \eta) M_{n, i} \\
& \times\left[\delta_{x_{0}, x_{1}}\right. \\
& -\frac{f_{r, n}^{2}(1+i \eta)}{f_{r, n}^{2}(1+i \eta)-f^{2}}\left(g_{\varepsilon_{n}} * g_{\varepsilon_{n}}\right) \\
& \left.\times\left(x_{0}-x_{1}\right) d x_{1}\right],
\end{aligned}
$$

where $\delta_{x_{0}, x_{1}}$ is the Kronecker delta and * means convolution with the argument $\left(x_{0}-x_{1}\right)$. It is seen that the transfer terms of the boundary impedance in Eq. (4) are proportional to $\left(g_{\varepsilon_{n}} * g_{\varepsilon_{n}}\right)\left(x_{0}-x_{1}\right)$. This function has been plotted in Fig. 4, and it can be observed that these transfer terms are largest close to $x_{1}$ and that the spatial memory in effect reaches $2 \varepsilon_{n}$ on either side of the response point $x_{1}$.

The total boundary impedance of the fuzzy substructure can be determined as the sum of all the impedances of all the sets, which yields

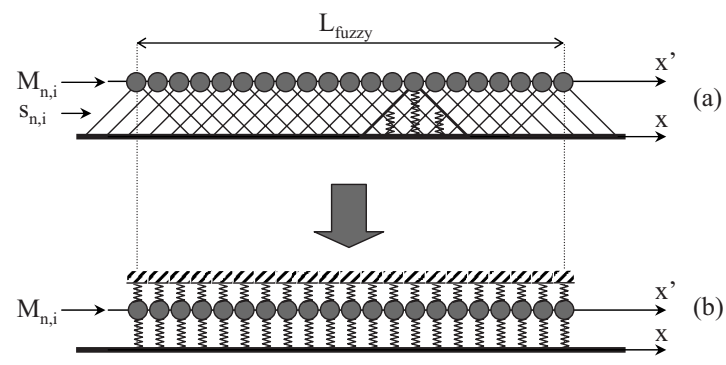

FIG. 3. A set of infinitely many identical oscillators attached to the master: (a) spatial oscillators and (b) equivalent local oscillators.

L. Friis and M. Ohlrich: Structural fuzzy with continuous boundary 


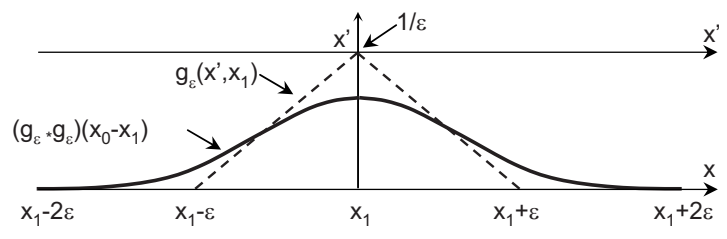

FIG. 4. Fuzzy oscillator with spatial coupling: ---, stiffness distribution function $g_{\varepsilon}\left(x^{\prime}-x_{1}\right)$ of the oscillator springs and -, convolution of the stiffness distribution with itself $\left(g_{\varepsilon} * g_{\varepsilon}\right)\left(x_{0}-x_{1}\right)$.

$$
\underline{z}_{\text {fuzzy }, \varepsilon}\left(x_{0}-x_{1}\right) d x_{1}=\sum_{n=1}^{\infty} z_{\varepsilon, n}\left(x_{0}-x_{1}, f_{r, n}\right) d x_{1} .
$$

This discrete sum can be replaced by an integral if there is sufficient modal overlap. ${ }^{20}$ This is done by replacing $z_{\varepsilon, n}\left(x_{0}\right.$ $\left.-x_{1}, f_{r, n}\right) d x_{1}$ from Eq. (4) with a continuous distribution denoted by $z_{\varepsilon}\left(x_{0}-x_{1}, f_{r}\right) d x_{1} d f_{r}$, where $f_{r}$ is a continuous frequency variable $\left(f_{r, n} \rightarrow f_{r}\right)$, and further by assuming that the sets resonate between bounding frequencies $f_{r \text {, lower }}$ and $f_{r \text {,upper. }}$ This gives

$$
\underline{z}_{\text {fuzzy }, \varepsilon}\left(x_{0}-x_{1}\right) d x_{1}=\int_{f_{r, \text { lower }}}^{f_{r, \text { upper }}} z_{\varepsilon}\left(x_{0}-x_{1}, f_{r}\right) d x_{1} d f_{r} .
$$

By substituting herein the detailed expression of a continuous version of Eq. (4), the fuzzy boundary impedance becomes

$$
\begin{aligned}
& \underline{Z}_{\text {fuzzy, },}\left(x_{0}-x_{1}\right) d x_{1} \\
& =-\frac{i 2 \pi f}{L_{\text {fuzzy }}} \int_{f_{r, \text { lower }}}^{f_{r \text {,upper }}}\left(\frac{f_{r}^{2}}{f^{2}}\right)(1+i \eta) m_{\text {fuzzy }}\left(f_{r}\right) \\
& \quad \times\left[\delta_{x_{0}, x_{1}}-\frac{f_{r}^{2}(1+i \eta)}{f_{r}^{2}(1+i \eta)-f^{2}}\left(g_{\varepsilon} * g_{\varepsilon}\right)\left(x_{0}\right.\right. \\
& \left.\left.-x_{1}\right) d x_{1}\right] d f_{r},
\end{aligned}
$$

where $M_{n, i}$ has been replaced by $m_{\text {fuzzy }}\left(f_{r}\right) d f_{r}$ that corresponds to the total mass of the fuzzy substructure resonating in the infinitesimal frequency band between $f_{r}$ and $f_{r}+d f_{r}$.

\section{Equivalent local modeling method}

A numerical implementation of the boundary impedance given in Eq. (7) is rather complicated due to its nonlocal nature and requires, for instance, the use of a finite element model with special fuzzy elements. Unfortunately, this is in contradiction with the idea of the theory of fuzzy structures being a simple modeling tool. However, as indicated in Fig. 3(b), Soize ${ }^{3}$ solved this problem by introducing a set of equivalent local oscillators that can imitate the boundary forces induced by a set of spatial oscillators. This means that the boundary impedance $\underline{z}_{\text {fuzzy, },}$ in Eq. (7) can be replaced with an equivalent boundary impedance $\underline{z}_{\text {fuzzy,equ }}$ that has direct terms only. Figure 3(b) shows that the equivalent local oscillator corresponds to a modified simple oscillator with a spring stiffness $\underline{s}_{1, n, i}$, but where the point mass is grounded via a second spring with stiffness $\underline{s}_{2, n, i}$, such that $\underline{s}_{n, i}=\underline{s}_{1, n, i}$ $+\underline{s}_{2, n, i}$. This means that the equivalent oscillator is springlike at low frequencies because the massless bar that supports the point mass in Fig. 1(a) is unable to rotate; the spatial oscillators, therefore, impose springlike properties on the master at low frequencies.

The relationship between the stiffnesses of the springs for the $n$th set of equivalent oscillators shown in Fig. 3(b) is given in terms of the equivalent coupling factor $\alpha_{n, i}$ and can be expressed as ${ }^{7}$

$$
\alpha_{n, i}=\underline{s}_{1, n, i} /\left(\underline{s}_{1, n, i}+\underline{s}_{2, n, i}\right)=\underline{s}_{1, n, i} \underline{s}_{n, i}
$$

where $\left.\left.\alpha_{n, i} \in\right] 0,1\right]$. This parameter $\alpha_{n, i}$ must be determined as a function of the characteristic dimension $\varepsilon_{n}$ of the spatial oscillators, and such a derivation is presented in Sec. III. By introducing $\alpha_{n, i}$, the boundary impedance $z_{\text {equ }, n}\left(x_{0}, f_{r, n}\right)$ of the $n$th set of equivalent local oscillators becomes ${ }^{3}$

$$
\begin{aligned}
\underline{z}_{\text {equ }, n}\left(x_{0}, f_{r, n}\right)= & \frac{\underline{s}_{n, i}}{i \omega}\left(1-\frac{\underline{S}_{n, i}}{\underline{S}_{n, i}-\omega^{2} M_{n, i}} \alpha_{n, i}\right) \\
= & -i \omega\left(\frac{f_{r, n}^{2}}{f^{2}}\right)(1+i \eta) M_{n, i} \\
& \times\left[1-\frac{f_{r, n}^{2}(1+i \eta)}{f_{r, n}^{2}(1+i \eta)-f^{2}} \alpha_{n, i}\right] .
\end{aligned}
$$

Note that $\alpha_{n, i} \rightarrow 1$ when $\underline{s}_{2, n, i} \rightarrow 0$ and $\underline{z}_{\text {equ, } n}$ will then approach the impedance of a simple oscillator. Further, when $\alpha_{n, i} \rightarrow 0$, then $\underline{s}_{1, n, i} \rightarrow 0$, which indicates that the structural fuzzy has no effect on the master. Inserting a continuous version of Eq. (9) into Eq. (6) yields the boundary impedance $\underline{z}_{\text {fuzzy,equ }}\left(x_{0}\right)$ of the equivalent fuzzy,

$$
\begin{aligned}
\underline{z}_{\text {fuzzy, equ }}\left(x_{0}\right)= & -\frac{i 2 \pi f}{L_{\text {fuzzy }}} \int_{f_{r, \text { lower }}}^{f_{r, \text { upper }}}\left(\frac{f_{r}^{2}}{f^{2}}\right)(1+i \eta) m_{\text {fuzzy }}\left(f_{r}\right) \\
& \times\left[1-\frac{f_{r}^{2}(1+i \eta)}{f_{r}^{2}(1+i \eta)-f^{2}} \alpha\right] d f_{r} .
\end{aligned}
$$

This expression also applies for two-dimensional structural fuzzy when the connection length $L_{\text {fuzzy }}$ is replaced by the connection area $A_{\text {fuzzy }}$. It should be noted that the equivalent coupling factor $\alpha$ generally is a function of frequency. In a companion paper, ${ }^{7}$ it was suggested that $\alpha$ can be determined as a function of the ratio $\varepsilon / \lambda$, where $\lambda$ is the wavelength of vibration in the master structure, which has one-dimensional wave motion only.

\section{METHOD OF DETERMINING THE EQUIVALENT COUPLING FACTOR}

\section{A. Matching of boundary forces}

Before the suggested equivalent modeling method can be utilized, it is necessary to establish a relationship between the parameters $\varepsilon$ and $\alpha$. This requires that the boundary forces induced by the set of spatial oscillators are matched with the forces induced by the equivalent local oscillators. Results for the equivalent coupling factor $\alpha$ determined in this way were published by Soize $^{3}$ but only for a very specific case of a simply supported beam with an attached fuzzy substructure with continuous boundary. His results show the mean value of $\alpha$ as a function of the spatial width $\varepsilon$ for only 
three coarse frequency bands with a width of $100 \mathrm{~Hz}$ going from 350 to $650 \mathrm{~Hz}$. The authors of the present paper, however, seek a simple and general method for finding $\alpha$. Accordingly, a method for determining $\alpha$ will be presented in the following, again as a function of the ratio $c=\varepsilon / \lambda$. For simple master structures with sinusoidal vibration, the wavelength $\lambda$ is equal to the free wavelength. For master structures with more complicated eigenfunctions, it is suggested that the term wavelength is replaced by twice the distance between adjacent nodes.

Next, the matching of the boundary forces induced by the $n$th set of spatial and equivalent oscillators can be expressed as

$$
\underline{F}_{\text {equ }, n}{ }^{\prime}\left(x_{0}\right)=\underline{F}_{\varepsilon, n}{ }^{\prime}\left(x_{0}\right) .
$$

In terms of velocities and impedances, this becomes

$$
\underline{z}_{\text {equ }, n}\left(x_{0}\right) \underline{v}\left(x_{0}\right)=\int_{L_{\text {fuzzy }}} \underline{z}_{\varepsilon, n}\left(x_{0}-x_{1}\right) \underline{v}\left(x_{1}\right) d x_{1} .
$$

Inserting herein the expressions for the impedances $\underline{z}_{\varepsilon, n}\left(x_{0}\right.$ $\left.-x_{1}\right)$ and $z_{\text {equ, } n}\left(x_{0}\right)$ from Eqs. (4) and (9), respectively, yields

$$
\begin{aligned}
\underline{s}_{n, i}(1 & \left.-\frac{\underline{s}_{n, i}}{\underline{s}_{n, i}-\omega^{2} M_{n, i}} \alpha\right) \underline{u}\left(x_{0}\right) \\
= & \underline{s}_{n, i}\left[\underline{u}\left(x_{0}\right)-\int_{L_{\text {fuzzy }}} \frac{\underline{s}_{n, i}}{\underline{s}_{n, i}-\omega^{2} M_{n, i}}\left(g_{\varepsilon} * g_{\varepsilon}\right)\right. \\
& \left.\times\left(x_{0}-x_{1}\right) \underline{u}\left(x_{1}\right) d x_{1}\right] .
\end{aligned}
$$

Next, by rearranging, we obtain an equation that has similar terms on each side of the equality sign,

$$
\begin{aligned}
& \underline{s}_{n, i}[\left.\underline{u}\left(x_{0}\right)-\frac{\underline{s}_{n, i}}{\underline{s}_{n, i}-\omega^{2} M_{n, i}} \alpha \underline{u}\left(x_{0}\right)\right] \\
& \quad=\underline{s}_{n, i}\left[\underline{u}\left(x_{0}\right)-\frac{\underline{s}_{n, i}}{\underline{s}_{n, i}-\omega^{2} M_{n, i}}\right. \\
&\left.\quad \times \int_{L_{\text {fuzzy }}}\left(g_{\varepsilon} * g_{\varepsilon}\right)\left(x_{0}-x_{1}\right) \underline{u}\left(x_{1}\right) d x_{1}\right] .
\end{aligned}
$$

By eliminating these terms, Eq. (14) is reduced to

$$
\alpha \underline{u}\left(x_{0}\right)=\int_{L_{\text {fuzzy }}}\left(g_{\varepsilon} * g_{\varepsilon}\right)\left(x_{0}-x_{1}\right) \underline{u}\left(x_{1}\right) d x_{1},
$$

from which an expression for $\alpha$ is obtained,

$$
\alpha\left(x_{0}\right)=\frac{\int_{L_{\text {fuzzy }}}\left(g_{\varepsilon} * g_{\varepsilon}\right)\left(x_{0}-x_{1}\right) \underline{u}\left(x_{1}\right) d x_{1}}{u\left(x_{0}\right)} .
$$

\section{B. Approximate expressions for the master's equivalent coupling factor}

Determining $\alpha$ from Eq. (16) requires a detailed knowledge about the form of the motion displacement $\underline{u}\left(x_{1}\right)$ of the master. At this point in the analysis, this form has not been identified and it is, therefore, preliminarily approximated by a suitably simple function of $x$. Moreover, since we are mostly concerned with prediction in the midfrequency range, it is assumed that the wavelength of the master vibration is shorter than the length $L$ of the structure. This also implies that the wave motion in the master is relatively independent of its boundary conditions. Therefore, a sinusoidal function is a good approximation for the one-dimensional vibration of the master, with the exception of the regions very close to the edges. Thus, it is assumed that the displacement $u\left(x_{1}\right)$ of the master can be described as

$$
\underline{u}\left(x_{1}\right)=\sin \left(\frac{2 \pi}{\lambda} x_{1}\right),
$$

where $\lambda$ is the free wavelength. ${ }^{21}$ Inserting this in Eq. (16) yields $\alpha$ as a function of $x_{0}$ and $c$,

$$
\alpha\left(x_{0}, c\right)=\frac{\int_{L_{\mathrm{fuzzy}}}\left(g_{\varepsilon} * g_{\varepsilon}\right)\left(x_{0}-x_{1}\right) \sin \left(\frac{2 \pi}{\lambda} x_{1}\right) d x_{1}}{\sin \left(\frac{2 \pi}{\lambda} x_{0}\right)} .
$$

Now, the convolution product inherent in Eq. (18) has only nonzero values when $x_{1} \in\left[\left(x_{0}-2 \varepsilon\right),\left(x_{0}+2 \varepsilon\right)\right]$ and it is, therefore, sufficient to solve the integral in this interval provided that $x_{1}$ is at least $2 \varepsilon$ from the edge of the fuzzy. If this is fulfilled, no truncation errors occur and an analytical solution of Eq. (18) is found by using the symbolic mathematics software MAPLE® (version 10),

$$
\alpha\left(x_{0}, c\right)=\alpha(c)=\left[\frac{\sin (\pi c)}{\pi c}\right]^{4} .
$$

This is a surprisingly simple result, which is independent of the position $x_{0}$ on the master structure because of the homogeneity of the fuzzy. The function $\sin (\pi c) /(\pi c)$ in Eq. (19) is recognized as the sink function. Figure 5 shows $\alpha$ as a function of $c=\varepsilon / \lambda$ for two different regions. In Fig. 5(a), it is seen that $\alpha=1$ when $\varepsilon=0$, which is the case of no spatial memory. Up to about $c=0.8, \alpha$ appears to be a uniformly descending function. For values higher than $c=0.8, \alpha$ becomes very small and the ordinate is, therefore, extended in Fig. 5(b). Here, the behavior of the sink function is easily recognized, showing soft minima and maxima, and it is revealed that $\alpha$ becomes zero when $c=1,2,3, \ldots$.

For the remaining part of the fuzzy where $x_{1}$ is closer than $2 \varepsilon$ from the edge of the fuzzy, it is not possible to integrate with respect to $x_{1}$ in the whole interval [ $\left(x_{0}\right.$ $\left.-2 \varepsilon),\left(x_{0}+2 \varepsilon\right)\right]$ and the solution for $\alpha$ becomes quite complicated. The simplest way to overcome this problem is to assume that $\alpha$ takes on a constant value that can be calculated from Eq. (19). The truncation error introduced because of this assumption naturally depends on the length $2 \varepsilon$. Hence, the larger the values of $\varepsilon$ are, the larger the introduced truncation error is.

At this point, two assumptions have been made: First, the vibration of the master is approximated by a sinusoidal function. Errors due to this assumption will only be significant at low frequencies where the free wavelength ${ }^{21}$ in the master is large. Second, $\alpha$ is considered to be constant with position. As mentioned, this assumption depends on the 

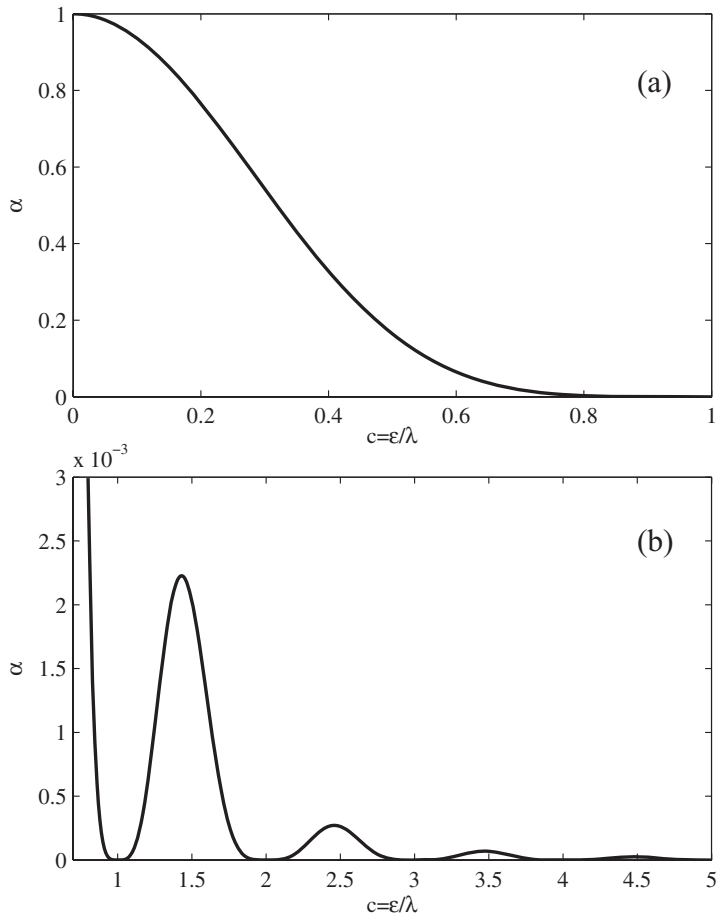

FIG. 5. Variation of the equivalent coupling factor $\alpha$ as a function of the ratio $c=\varepsilon / \lambda$. (a) Values of $c$ from 0 to 1 and (b) from 0.8 to 5 .

variation of $\varepsilon$ with frequency. The significance of these errors in the prediction of $\alpha$ will be examined in Sec. V, where numerical simulations will be presented for a plate master structure with an attached fuzzy substructure with spatial memory.

\section{STRUCTURAL FUZZY WITH TWO-DIMENSIONAL SPATIAL MEMORY}

\section{A. Determination of the two-dimensional fuzzy boundary impedance}

So far, the method of including spatial memory has been restricted to fuzzy substructures attached to the master structure through a one-dimensional boundary. Most real-life fuzzy structures, however, are attached to their master through a surface that also undergoes two-dimensional vibration. Therefore, the method of including spatial memory in this modeling of fuzzy structures is required and it is, therefore, extended to two dimensions in the following.

To accomplish this, the stiffness distribution function $g_{\varepsilon}\left(x^{\prime}-x_{1}\right)$ in Eq. (3) for the spatial oscillator is initially replaced by a two-dimensional version $g_{\varepsilon}\left(\left|r^{\prime}, \theta^{\prime} ; r_{1}, \theta_{1}\right|\right)$, which is a function of the distance $\left|r^{\prime}, \theta^{\prime} ; r_{1}, \theta_{1}\right|$ between two surface points $\left(r^{\prime}, \theta^{\prime}\right)$ and $\left(r_{1}, \theta_{1}\right)$ described in polar coordinates; it should be noted that the point mass of the spatial oscillator is located at $\left(r^{\prime}, \theta^{\prime}\right)$. This two-dimensional stiffness distribution that is shown in Fig. 6(a) in a Cartesian coordinate system represents a cone with a radius of $\varepsilon$ at its base and a volume of 1 . Based on these requirements, the distribution can be expressed mathematically as

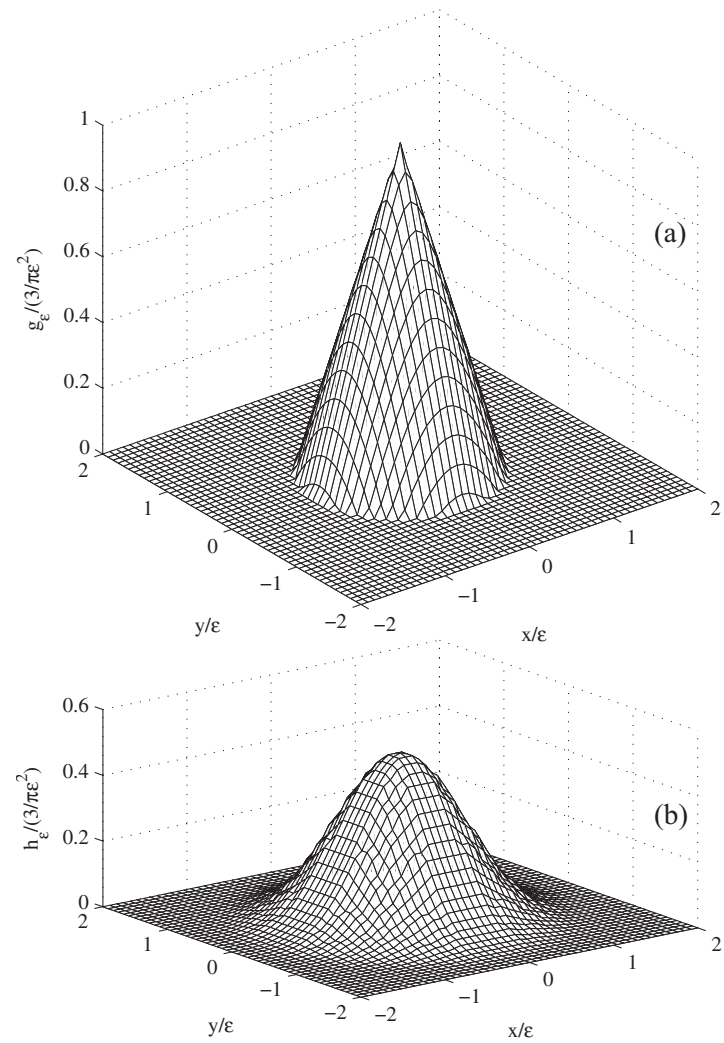

FIG. 6. (a) Normalized stiffness density distribution $g_{\varepsilon}$ of the oscillator springs. (b) Variation of the function $h_{\varepsilon}$ with $x$ and $y$.

$$
\begin{aligned}
g_{\varepsilon}\left(\left|r^{\prime}, \theta^{\prime} ; r_{1}, \theta_{1}\right|\right)= & \frac{\varepsilon-\left|r^{\prime}, \theta^{\prime} ; r_{1}, \theta_{1}\right|}{\frac{1}{3} \pi \varepsilon^{3}} 1_{\left[\left|r^{\prime}, \theta^{\prime} ; r_{1}, \theta_{1}\right| \leqslant \varepsilon\right]} \\
= & \frac{\varepsilon-\sqrt{r^{\prime 2}+r_{1}^{2}-2 r^{\prime} r_{1} \cos \left(\theta^{\prime}-\theta_{1}\right)}}{\frac{1}{3} \pi \varepsilon^{3}} \\
& \times 1_{\left[\left|r^{\prime}, \theta^{\prime} ; r_{1}, \theta_{1}\right| \leqslant \varepsilon\right]},
\end{aligned}
$$

where $1_{\left[\left|r^{\prime}, \theta^{\prime} ; r_{1}, \theta_{1}\right| \leqslant \varepsilon\right]}$ is a function that is unity when $\left|r^{\prime}, \theta^{\prime} ; r_{1}, \theta_{1}\right| \leqslant \varepsilon$, and 0 elsewhere. The corresponding stiffness distribution of a two-dimensional spatial oscillator $\underline{s}_{\varepsilon, n, i}\left(\left|r^{\prime}, \theta^{\prime} ; r_{1}, \theta_{1}\right|\right)$, thus, becomes

$$
\begin{aligned}
\underline{s}_{\varepsilon, n, i}\left(\left|r^{\prime}, \theta^{\prime} ; r_{1}, \theta_{1}\right|\right) & =\underline{s}_{n, i} g_{\varepsilon}\left(\left|r^{\prime}, \theta^{\prime} ; r_{1}, \theta_{1}\right|\right) \\
& =\left(M_{n, i} \omega_{r, n}^{2}\right)(1+i \eta) g_{\varepsilon}\left(\left|r^{\prime}, \theta^{\prime} ; r_{1}, \theta_{1}\right|\right)
\end{aligned}
$$

where it applies that $M_{n, i}=M_{n} / A_{\text {fuzzy }}$ and $A_{\text {fuzzy }}$ is the area of the fuzzy connection surface. In the case of a onedimensional connection boundary, Ref. 7 gives an expression for the force per unit length $\underline{F}_{n, i}{ }^{\prime}\left(x_{0}\right)$ at $x_{0}$ due to the displacement $\underline{u}\left(x_{1}\right)$ at $x_{1}$ caused by only one spatial oscillator. By replacing this one-dimensional version of $g_{\varepsilon}$ by the new two-dimensional version, we obtain an expression for the relationship between the force per unit area $\underline{F}_{n, i}{ }^{\prime \prime}\left(r_{0}, \theta_{0}\right)$ and the displacement $u\left(r_{1}, \theta_{1}\right)$ that reads 


$$
\begin{aligned}
\underline{F}_{n, i}^{\prime \prime}\left(r_{0}, \theta_{0}\right)= & \underline{s}_{\varepsilon, n, i}\left(\left|r^{\prime}, \theta^{\prime} ; r_{1}, \theta_{1}\right|\right)\left[\delta_{\left(r^{\prime}, \theta^{\prime}\right),\left(r_{1}, \theta_{1}\right)}\right. \\
& \left.-\frac{\underline{s}_{\varepsilon, n, i}\left(\left|r^{\prime}, \theta^{\prime} ; r_{1}, \theta_{1}\right|\right) r_{1} d r_{1} d \theta_{1}}{-\omega^{2} M_{n, i}+\underline{s}_{n, i}}\right] \underline{u}\left(r_{1}, \theta_{1}\right),
\end{aligned}
$$

where the infinitesimal area $d A_{1}$ is given as $d A_{1}=r_{1} d r_{1} d \theta_{1}$. Next, to find the force per unit area $\underline{F}_{n}{ }^{\prime \prime}\left(r_{0}, \theta_{0}\right)$ due to a set of infinitely many identical spatial oscillators with the base displacement $\underline{u}\left(r_{1}, \theta_{1}\right)$, Eq. (22) is integrated with respect to $\left(r^{\prime}, \theta^{\prime}\right)$ over the fuzzy connection surface as

$$
\begin{aligned}
\underline{F}_{n}^{\prime \prime}\left(r_{0}, \theta_{0}\right)= & \int_{A_{\text {fuzzy }}} \underline{s}_{\varepsilon, n, i}\left(\left|r^{\prime}, \theta^{\prime} ; r_{1}, \theta_{1}\right|\right)\left[\delta_{\left(r^{\prime}, \theta^{\prime}\right),\left(r_{1}, \theta_{1}\right)}\right. \\
& \left.-\frac{\underline{S}_{\varepsilon, n, i}\left(\left|r^{\prime}, \theta^{\prime} ; r_{1}, \theta_{1}\right|\right) d A_{1}}{-\omega^{2} M_{n, i}+\underline{s}_{n, i}}\right] \underline{u}\left(r_{1}, \theta_{1}\right) d A^{\prime} .
\end{aligned}
$$

Finally, this expression can be reduced to

$$
\begin{aligned}
\underline{F}_{n}^{\prime \prime}\left(r_{0}, \theta_{0}\right)= & {\left[\underline{s}_{n, i} \delta_{\left(r^{\prime}, \theta^{\prime}\right),\left(r_{1}, \theta_{1}\right)}-\frac{\underline{s}_{n, i}^{2}}{-\omega^{2} M_{n, i}+\underline{s}_{n, i}}\right.} \\
& \left.\times h_{\varepsilon}\left(\left|r_{1}, \theta_{1} ; r_{0}, \theta_{0}\right|\right) d A_{1}\right] \underline{u}\left(r_{1}, \theta_{1}\right),
\end{aligned}
$$

where the function $h_{\varepsilon}\left(\left|r_{1}, \theta_{1} ; r_{0}, \theta_{0}\right|\right)$ is given by

$$
\begin{aligned}
& h_{\varepsilon}\left(\left|r_{1}, \theta_{1} ; r_{0}, \theta_{0}\right|\right) \\
& \quad=\int_{A_{\text {fuzzy }}} g_{\varepsilon}\left(\left|r^{\prime}, \theta^{\prime} ; r_{1}, \theta_{1}\right|\right) g_{\varepsilon}\left(\left|r^{\prime}, \theta^{\prime} ; r_{0}, \theta_{0}\right|\right) d A^{\prime} \\
& \quad=\int_{0}^{2 \pi} \int_{0}^{2 \varepsilon} g_{\varepsilon}\left(\left|r^{\prime}, \theta^{\prime} ; r_{1}, \theta_{1}\right|\right) g_{\varepsilon}\left(\left|r^{\prime}, \theta^{\prime} ; r_{0}, \theta_{0}\right|\right) r^{\prime} d r^{\prime} d \theta^{\prime} .
\end{aligned}
$$

It follows that the boundary impedance of the $n$th set multiplied by $d A_{1}$ is given as

$$
\begin{aligned}
\underline{z}_{\varepsilon, n}( & \left.\left|r_{0}, \theta_{0} ; r_{1}, \theta_{1}\right|\right) d A_{1} \\
= & \frac{\underline{s}_{n, i}}{i \omega}\left[\delta_{\left(r^{\prime}, \theta^{\prime}\right),\left(r_{1}, \theta_{1}\right)}-\frac{\underline{s}_{n, i}}{-\omega^{2} M_{n, i}+\underline{s}_{n, i}}\right. \\
& \left.\times h_{\varepsilon}\left(\left|r_{1}, \theta_{1} ; r_{0}, \theta_{0}\right|\right) d A_{1}\right],
\end{aligned}
$$

and by analogy to Eq. (7), the fuzzy boundary impedance becomes

$$
\begin{aligned}
& \underline{z}_{\text {fuzzy }, \varepsilon}\left(\left|r_{0}, \theta_{0} ; r_{1}, \theta_{1}\right|\right) d A_{1} \\
&=-\frac{i 2 \pi f}{A_{\text {fuzzy }}} \int_{f_{r, \text { lower }}}^{f_{r, \text { upper }}}\left(\frac{f_{r}^{2}}{f^{2}}\right)(1+i \eta) m_{\text {fuzzy }}\left(f_{r}\right) \\
& \quad \times\left[\delta_{\left(r^{\prime}, \theta^{\prime}\right),\left(r_{1}, \theta_{1}\right)}-\frac{f_{r}^{2}(1+i \eta)}{f_{r}^{2}(1+i \eta)-f^{2}}\right. \\
&\left.\quad \times h_{\varepsilon}\left(\left|r_{1}, \theta_{1} ; r_{0}, \theta_{0}\right|\right) d A_{1}\right] d f_{r} .
\end{aligned}
$$

From Eq. (27), it is seen that transfer impedance terms of the fuzzy substructure are proportional to the function $h_{\varepsilon}\left(\left|r_{1}, \theta_{1} ; r_{0}, \theta_{0}\right|\right)$. This function can be calculated numerically and the result is shown in Fig. 6(b) for the case $\left(r_{1}, \theta_{1}\right)=(0,0)$. It is clearly observed that $h_{\varepsilon}$ looks similar to a two-dimensional version of the convolution shown in Fig. 4. Also, it can be seen that $h_{\varepsilon}$ extends to a radius of $2 \varepsilon$ relative to $\left(r_{1}, \theta_{1}\right)$ and that the volume under the surface is unity.

\section{B. Determination of the equivalent coupling factor}

By analogy to the method in Sec. III, the equivalent coupling factor will now be determined as a function of $c$ $=\varepsilon / \lambda_{b}$, where $\lambda_{b}$ is the vibration wavelength for bending waves. Again, $\lambda_{b}$ is suggested to be the free wavelength for simple master structures and twice the distance between adjacent nodes for more complicated master structures. An expression for the equivalent coupling factor is found by extending the expression in Eq. (16) to two dimensions yielding

$$
\begin{aligned}
\alpha\left(r_{0}, \theta_{0} ; c\right) & =\frac{\int_{A_{\text {fuzzy }}} h_{\varepsilon}\left(\left|r_{1}, \theta_{1} ; r_{0}, \theta_{0}\right|\right) \underline{u}\left(r_{1}, \theta_{1}\right) d A_{1}}{u\left(r_{0}, \theta_{0}\right)} \\
& =\frac{\int_{0}^{2 \pi} \int_{0}^{2 \varepsilon} h_{\varepsilon}\left(\left|r_{1}, \theta_{1} ; r_{0}, \theta_{0}\right|\right) \underline{u}\left(r_{1}, \theta_{1}\right) r_{1} d r_{1} d \theta_{1}}{\underline{u}\left(r_{0}, \theta_{0}\right)} .
\end{aligned}
$$

As for the one-dimensional case, the determination of $\alpha$ requires prior knowledge of the vibration displacements of the master structure. Again this problem is overcome by approximating the displacements by a product of two sinusoidals as

$$
\underline{u}\left(x_{1}, y_{1}\right)=\sin \left(\frac{2 \pi}{\lambda_{x}} x_{1}\right) \sin \left(\frac{2 \pi}{\lambda_{y}} y_{1}\right)
$$

where $\lambda_{x}$ and $\lambda_{y}$ are the vibration wavelengths for bending motion in the $x$ and $y$ directions, respectively. By substituting $x_{1}=r_{1} \cos \left(\theta_{1}\right)$ and $y_{1}=r_{1} \sin \left(\theta_{1}\right)$ in Eq. (29), the displacement is transformed to polar coordinates, giving

$$
\underline{u}\left(r_{1}, \theta_{1}\right)=\sin \left[\frac{2 \pi}{\lambda_{x}} r_{1} \cos \left(\theta_{1}\right)\right] \sin \left[\frac{2 \pi}{\lambda_{y}} r_{1} \sin \left(\theta_{1}\right)\right] .
$$

Further, the bending wavelength $\lambda_{b}$ can be found from the relation between the wave numbers, ${ }^{21}$

$$
k_{b}^{2}=k_{x}^{2}+k_{y}^{2} \Leftrightarrow\left(\frac{2 \pi}{\lambda_{b}}\right)^{2}=\left(\frac{2 \pi}{\lambda_{x}}\right)^{2}+\left(\frac{2 \pi}{\lambda_{y}}\right)^{2},
$$

which by rearranging becomes

$$
\lambda_{b}=\sqrt{\frac{\lambda_{x}^{2} \lambda_{y}^{2}}{\lambda_{x}^{2}+\lambda_{y}^{2}}} .
$$

As for the one-dimensional case, it is suggested that $\lambda_{b}$ is replaced by the free wavelength for simple master structures and by twice the distance between adjacent nodes for master structures with more complicated eigenfunctions. Again, by only considering positions on the fuzzy connection surface that are at least a distance of $2 \varepsilon$ from the edges of the fuzzy, 

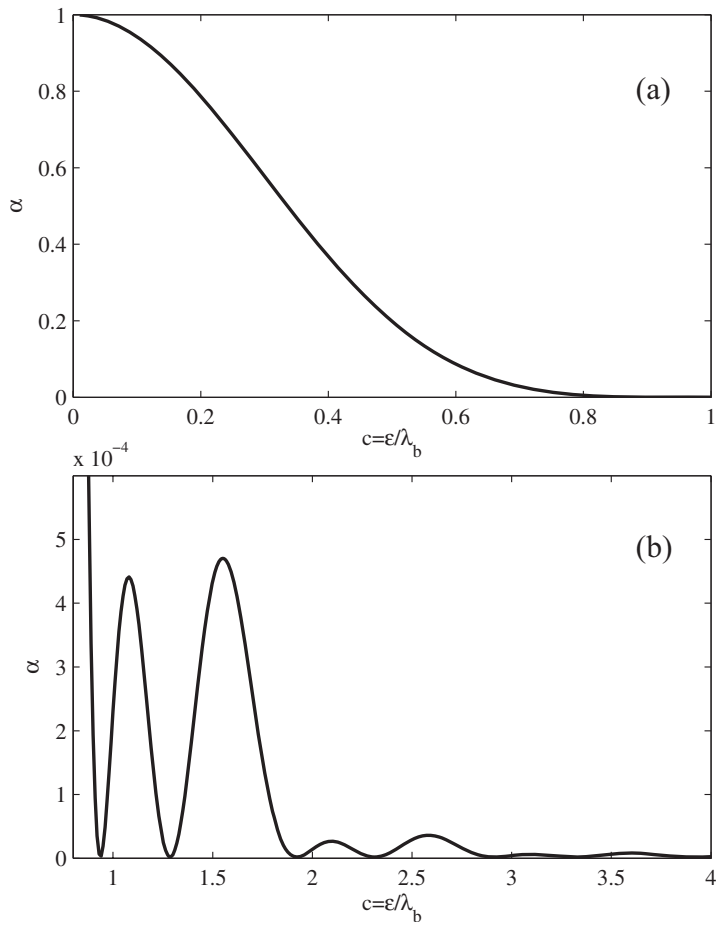

FIG. 7. Variation of the equivalent coupling factor $\alpha$ as a function of $c$ $=\varepsilon / \lambda_{b}$. (a) Values of $c$ from 0 to 1 and (b) from 0.8 to 4 .

an expression for $\alpha$ is found by substituting Eqs. (25) and (30) into Eq. (28). This expression becomes quite complicated and the integrals cannot be solved analytically. Nevertheless, by using numerical integration, $\alpha$ has been determined as a function of $c=\varepsilon / \lambda_{b}$ and the results are shown in Fig. 7.

First, it should be noted that $\alpha$ does not depend on the forcing position $\left(r_{0}, \theta_{0}\right)$ as long as the response position $\left(r_{1}, \theta_{1}\right)$ is at least a distance of $2 \varepsilon$ from the edges of the fuzzy connection surface. Second, it is found that $\alpha$ is insensitive to the specific values of $\lambda_{x}$ and $\lambda_{y}$ and only depends on $\lambda_{b}$. Figure 7(a) shows that the variation of $\alpha$ resembles the uniformly descending function seen in Fig. 5(a) for values up to $c=0.8$. Again, for values above $c=0.8$, the equivalent coupling factor takes on very small values. Closer inspection of this low value region [see Fig. 7(b)] reveals a different pattern of smooth minima and maxima from that observed in Fig. 5(b). Nevertheless, the equivalent coupling factor still becomes 0 for certain values of $c$.

\section{NUMERCIAL VALIDATION OF EQUIVALENT MODELING METHOD}

In a companion paper, ${ }^{7}$ it was shown that the spatial memory in the structural fuzzy significantly reduces its damping effect. This finding was achieved by using the equivalent modeling method just described in Sec. II. In the following, the equivalent modeling method will be validated for certain two-dimensional problems by numerical simulations. The finite element method ${ }^{22}$ has been used to solve the flexural vibration response of a rectangular plate that is considered as the master; the plate undergoes two-dimensional bending vibration and is assumed to be simply supported along all four edges that have side lengths $L_{x}$ and $L_{y}$, with an

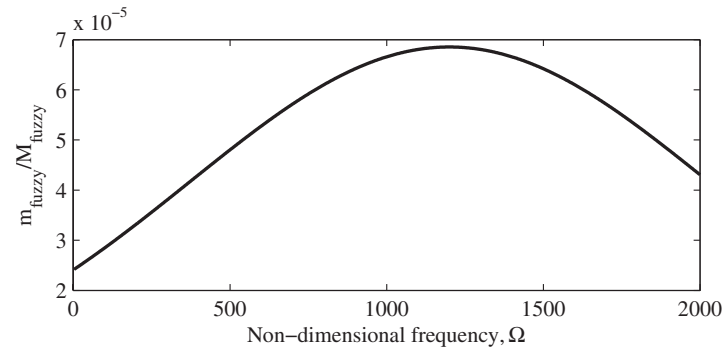

FIG. 8. Normalized resonating mass per unit frequency described by a normal distribution with a centre frequency $f_{r 0}$ corresponding to $\Omega=1200$ and a standard deviation $\mathrm{std}=0.7 f_{r 0}$.

aspect ratio given by $L_{y}=1.3 L_{x}$. A fuzzy substructure is attached to the plate on the whole surface area $A=L_{x} L_{y}$, so that $A_{\text {fuzzy }}=A$. The loss factor of the plate is set to 0.005 , whereas the loss factor of the fuzzy oscillator springs has been chosen to be 0.03 . The resonating mass per unit frequency $m_{\text {fuzzy }}\left(f_{r}\right)$ is a normal distribution given as

$$
m_{\text {fuzzy }}\left(f_{r}\right)=\frac{M_{\text {fuzzy }}}{\operatorname{std} \cdot \sqrt{2 \pi}} e^{-\left(f_{r 0}-f_{r}\right)^{2} /\left(2 \cdot \text { std }^{2}\right)},
$$

where $f_{r 0}$ is the center frequency and std is the standard deviation. For this distribution, the bounding frequencies $f_{r, \text { lower }}$ and $f_{r \text {,upper }}$ corresponds to $\Omega=0$ and $\infty$, respectively. The chosen mass distribution is shown in Fig. 8 as a function of the plate's nondimensional frequency $\Omega$ defined as

$$
\Omega=\omega \sqrt{\frac{12 \rho\left(1-\nu^{2}\right)}{E}} \frac{\sqrt{L_{x}^{2}+L_{y}^{2}}}{h},
$$

where $h$ is the plate thickness, and $\rho, E$, and $\nu$ are the density, Young's modulus, and Poisson's ratio of the plate, respectively. The center frequency $f_{r 0}$ corresponds to $\Omega=1200$ and the standard deviation $\mathrm{std}=0.7 f_{r 0}$. The total mass of the attached fuzzy $M_{\text {fuzzy }}$ is one-twentieth of the plate mass, $\rho A h$. Also, the free bending wavelength $\lambda_{b}$ in the master plate is found $^{21}$

$$
\lambda_{b}=2 \pi \sqrt{\frac{L_{x}^{2}+L_{y}^{2}}{\Omega}} .
$$

For the following response prediction, the boundary impedance of the fuzzy is computed by numerical integration of the integrals in Eqs. (27) and (10), where $L_{\text {fuzzy }}$ is replaced by $A_{\text {fuzzy }}$ in the latter. Further, $\varepsilon=c \lambda_{b}$ is assumed to be constant with frequency implying that $c$ and $\alpha$, respectively, increases and decreases with frequency.

First examined is the effect of a simple fuzzy without spatial memory that is modeled by using Eq. (27) with $\varepsilon$ $=0$. Figure 9 shows results for the vibration velocity response per unit harmonic force of the simply supported master plate, with and without such a fuzzy substructure. Here, the response location $(x, y)$ and excitation position $\left(x_{0}, y_{0}\right)$ coincide, so the results represent the direct mobility of the system. Since the fuzzy has no spatial memory, it is clearly seen that it has a strong damping effect on the vibration response of the master. This effect mainly occurs from $\Omega$ $=500$ and upwards, where the resonating mass per unit frequency has an appreciable value; that is, it is approximately 

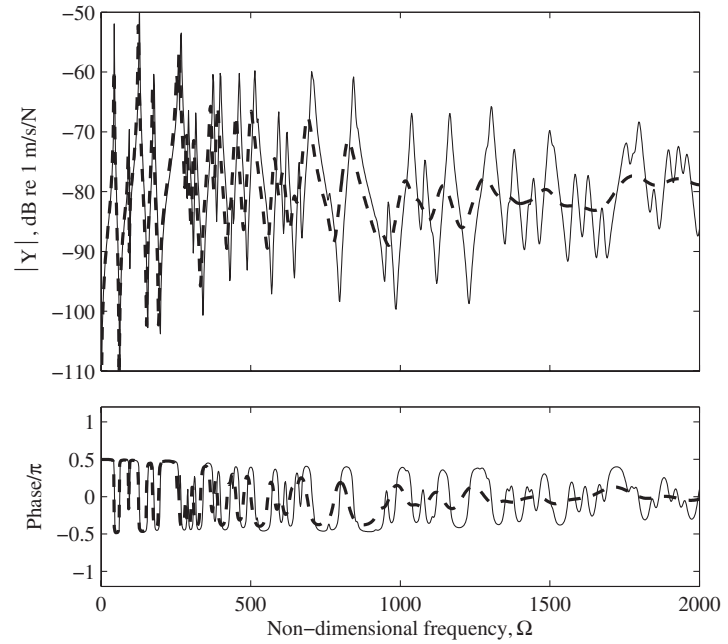

FIG. 9. Vibration velocity response per unit harmonic force of a simply supported plate, $\underline{Y}\left(x, y ; x_{0}, y_{0}\right)=\underline{v}(x, y) / \underline{F}\left(x_{0}, y_{0}\right)$, at $(x, y)=\left(x_{0}, y_{0}\right)$ $=\left(0.175 L_{x}, 0.5525 L_{x}\right)$. Curves: - without structural fuzzy and ---, with structural fuzzy without spatial memory using Eq. (27) with $\varepsilon=0$.

half or more of its maximum value according to the results in Fig. 8. At frequencies above $\Omega=1000$, it is furthermore seen that the direct mobility closely approaches the asymptote of $-80 \mathrm{~dB}$ for a master plate of infinite size. It should be noted that such high damping effect only occurs at early and moderate times for fuzzy substructures consisting of a finite number of oscillators, which are excited by an impulse, since the "absorbed" energy returns from the fuzzy to the master at later times. ${ }^{15,16}$ Drexel and Ginsberg ${ }^{23}$ also investigated this damping effect for a master cantilever beam with a finite number of spatially distributed discrete oscillators.

An example of the transfer mobility of the plate with and without structural fuzzy is shown in Fig. 10. As in Fig. 9, the vibration velocity response of the master is reduced significantly due to the fuzzy from about $\Omega=500$ and upwards. Also, at high frequencies, the response is strongly reduced and is even less than the transfer mobility of a corresponding infinitely large plate which has a low value of $-94 \mathrm{~dB}$ at $\Omega$ $=2000$.
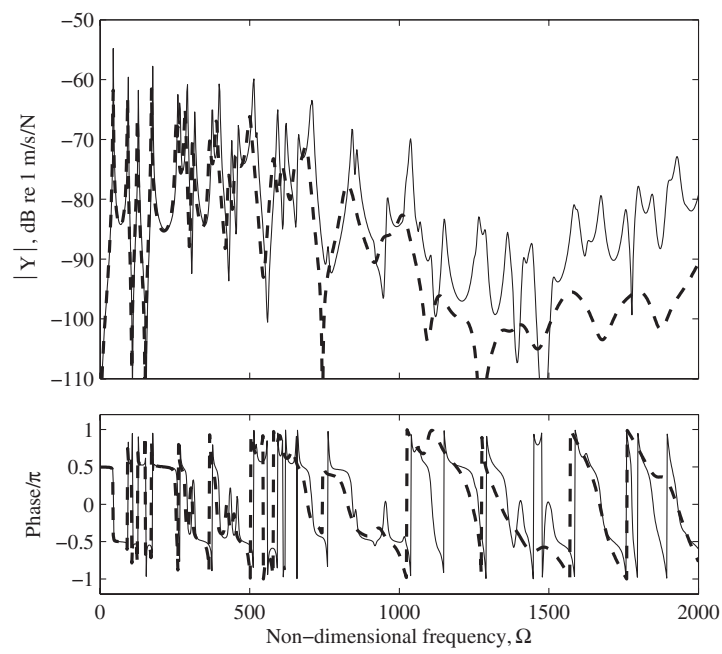

FIG. 10. As in Fig. 9, but for response at $(x, y)=\left(0.575 L_{x}, 1.1375 L_{x}\right)$ and excitation at $\left(x_{0}, y_{0}\right)=\left(0.175 L_{x}, 0.5525 L_{x}\right)$.
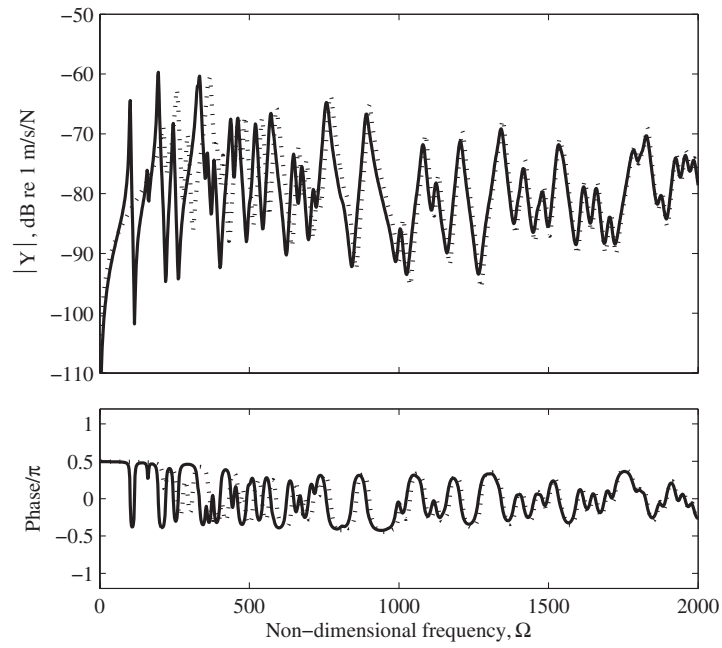

FIG. 11. Vibration velocity response per unit harmonic force of a simply supported plate, $\underline{Y}\left(x, y ; x_{0}, y_{0}\right)=\underline{v}(x, y) / \underline{F}\left(x_{0}, y_{0}\right)$, at $(x, y)=\left(x_{0}, y_{0}\right)$ $=\left(0.175 L_{x}, 0.5525 L_{x}\right)$. Conditions: Structural fuzzy with $\varepsilon=0.2 L_{x}$ and modeled by -, spatial oscillators using Eq. (27) and $\cdots \cdots \cdots \cdot$, equivalent local oscillators using Eq. (10).

Next presented is the validation of the equivalent prediction method. This validation consists in a comparison of simulated responses based on the prediction method using the equivalent local oscillators [Eq. (10)] and the reference method based on spatial oscillators [Eq. (27)]. Consider the simply supported master plate with an attached structural fuzzy that has a constant and high spatial memory $\varepsilon=0.2 L_{x}$. By using this value of $\varepsilon$ in Eq. (28), the corresponding equivalent coupling factor is found as a function of frequency and substituted in Eq. (10). A comparison of the two predictions is shown in Fig. 11 that displays the vibration responses in terms of the direct mobility. This reveals that there is a very good agreement between the two prediction methods from about $\Omega=500$ and upwards. Significant deviations occur only at low frequencies because of the assumed sinusoidal vibration [Eq. (29)]. Since the plate's edges are simply supported, the sinusoidals are only a really good approximation around the plate's natural frequencies; that is, the frequencies where the conditions $2 L / \lambda_{x}=1,2,3, \ldots$, and $2 L / \lambda_{y}=1,2,3, \ldots$, are both fulfilled. Nevertheless, this error in the estimation of $\alpha$ rapidly reduces with increasing frequency. Evidently, truncation errors that occur at all frequencies due to the constant value of $\varepsilon$ do not have a large influence on the prediction based on equivalent oscillators. Moreover, compared to the previous case of no spatial memory (Fig. 9), it is also observed that the spatial memory drastically reduces the damping effect of the fuzzy. In the companion paper, ${ }^{7}$ it was shown how the damping effect decreases when $\alpha$ is reduced. The reason for this is that a reduction in $\alpha$ corresponds to an increase in $\varepsilon$. Thus, due to the local angular motion in the master structure, the spring elements in the spatial oscillators counteract one another when $\varepsilon>0$.

The corresponding transfer mobility of the master plate with structural fuzzy of high spatial memory is shown in Fig. 12. Again, the two predictions are seen to be in good agree-

L. Friis and M. Ohlrich: Structural fuzzy with continuous boundary 

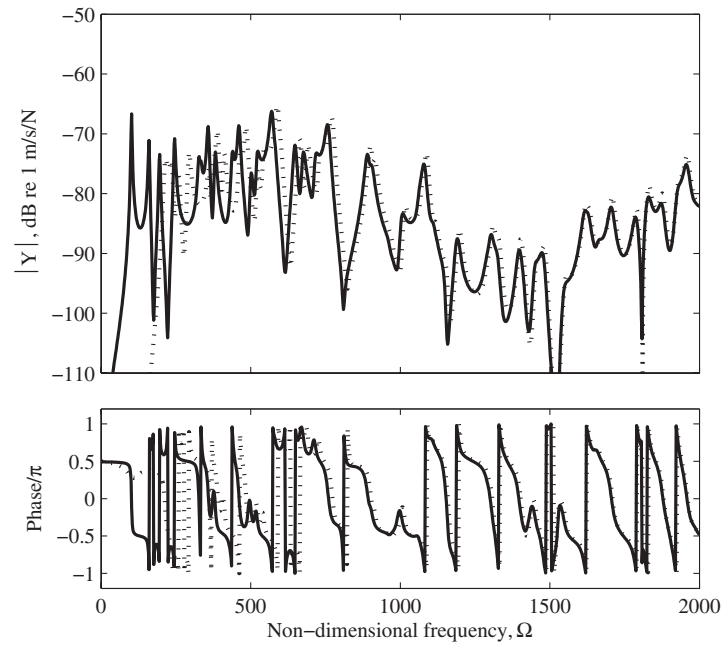

FIG. 12. As in Fig. 11, but for response at $(x, y)=\left(0.575 L_{x}, 1.1375 L_{x}\right)$ and excitation at $\left(x_{0}, y_{0}\right)=\left(0.175 L_{x}, 0.5525 L_{x}\right)$.

ment at frequencies from $\Omega=500$ and upwards. Furthermore, the spatial memory in the fuzzy here is also seen to radically reduce the damping effect.

The equivalent method of prediction performs poorly at low frequencies, say, below $\Omega=500$, because the sinefunction approximations of the master vibration pattern have to hold for two dimensions. For a one-dimensional master structure, however, this approximation only involves one direction, and the equivalent method is, therefore, expected to perform well also at lower frequencies. To demonstrate this, a beam with one-dimensional wave motion is considered as the master. A fuzzy substructure is attached on the whole length $L$ of the beam, so that $L_{\text {fuzzy }}=L$. The loss factor of the beam is set to 0.005 , whereas the loss factor of the fuzzy oscillator springs has been chosen to be 0.03 . The resonating mass per unit frequency is again a normal distribution where the center frequency $f_{r 0}$ corresponds to $\Omega=1200$ and std $=0.27 f_{r 0}$. The nondimensional frequency $\Omega$ for the beam is defined as

$$
\Omega=\omega \sqrt{\frac{12 \rho}{E}} \frac{L^{2}}{h} .
$$

In this example, the spatial memory $\varepsilon=0.1 L$ and the total mass of the fuzzy $M_{\text {fuzzy }}$ is one-twentieth of the beam mass, $\rho S L$, where $S$ is its cross-sectional area. The vibration velocity of the beam in terms of the input mobility is shown in Fig. 13. It is seen that the results of the two types of predictions given by Eqs. (7) and (10) are almost coinciding from $\Omega=250$ and upwards. Thus, as was anticipated, the equivalent method is found to apply at lower frequencies in the one-dimensional case. The results show that the prediction is reliable at least one octave lower in frequency for the twodimensional case.

\section{SUMMARY AND DISCUSSION}

In 1993, Soize ${ }^{3}$ introduced a method for modeling structural fuzzy with a continuous boundary. This method was later extended ${ }^{17}$ and validated. ${ }^{18}$ Moreover, a part of this method was systematically examined and reformulated in a
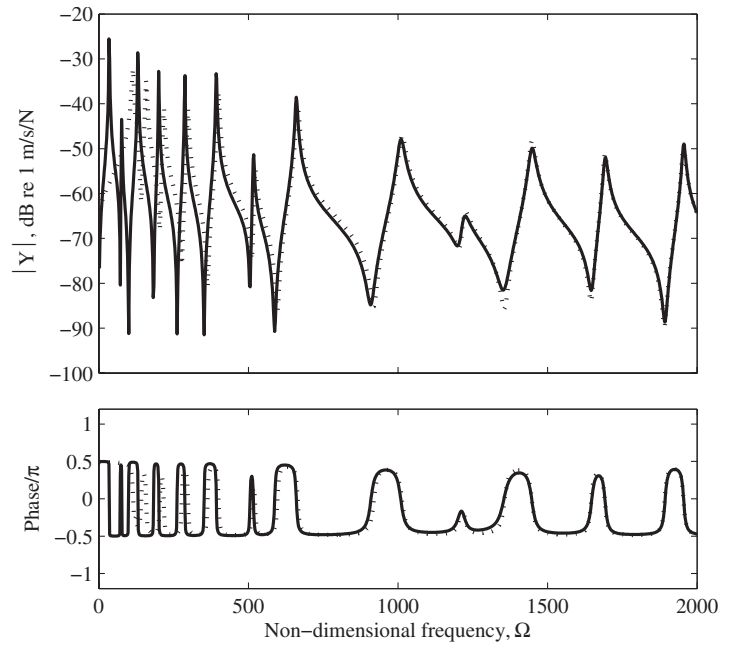

FIG. 13. Vibration velocity response per unit harmonic force of a simply supported beam, $\underline{Y}\left(x, x_{0}\right)=\underline{v}(x) / \underline{F}\left(x_{0}\right)$, at $x=x_{0}=0.445 L$. Conditions: Structural fuzzy with $\varepsilon=0.1 L$ modelled by - , spatial oscillators using Eq. (7) and $\cdots \cdots \cdots$, equivalent local oscillators using Eq. (10).

more simple form in a companion paper. ${ }^{7}$ The main objective of the present paper is to extend the method to two dimensions and to improve its usability.

Structural fuzzy with a continuous boundary can be modeled by including the stiffness of the fuzzy in terms of a spatial memory. Soize implemented such spatial memory by introducing the so-called spatial oscillators. However, to make the implementation of the fuzzy boundary impedance viable, he replaced these nonlocal spatial oscillators with $l o$ cal equivalent oscillators that can imitate boundary forces imposed on the master. This approximation required the introduction of the equivalent coupling factor that describes the relationship between the width of the spatial oscillators and the stiffness of the local equivalent oscillators. The current paper has presented a simple and general method for determining this factor as a function of a practical parameter given by the ratio between the width of the spatial oscillators and the free structural wavelength in the master structure. By assuming that the vibration pattern of the master structure can be approximated by one or more sinusoidals and that truncation effects at the end of the fuzzy connection boundary can be ignored, an expression for the equivalent coupling factor has been derived. This expression was evaluated analytically for one-dimensional wave motion in the master structure, and it is revealed that the solution is a very simple expression in the form of a sink function to the power of 4 .

For instructive reasons, the method of including memory in the structural fuzzy was originally formulated for a structural fuzzy attached to the master through a one-dimensional boundary. In the present paper, this formulation has been extended to two dimensions, so that it applies for structural fuzzy attached to the master through an area. This is achieved by introducing a two-dimensional stiffness distribution for the spatial oscillator. Additionally, an expression for the equivalent coupling factor is derived and computed numerically as a function of a practical parameter, that is, the ratio between spatial memory and the free wavelength of the 
vibration in the master. This solution proved to be closely related to the solution for one-dimensional wave motion in the master.

A validation of the equivalent method by using local equivalent oscillators as a replacement for the spatial oscillators has not previously been published in open literature. In the present paper, the validity of the method has, therefore, been tested by comparing numerical simulations of the response of a master plate with attached structural fuzzy. Results, based on the use of spatial oscillators and equivalent oscillators show a very good agreement for frequencies above $\Omega=500$, where $\Omega$ is the nondimensional frequency of the master structure. Below this frequency, errors are mainly caused by the assumption of sinusoidal vibration displacement. For a master beam with one-dimensional wave motion and a fuzzy substructure attached on the whole length, it is revealed that good agreement between predictions is already achieved from about $\Omega=250$. The reason is that the assumption of sinusoidal vibration displacement only has to be fulfilled for one dimension.

The present paper has made various assumptions in order to develop a viable method for modelling structural fuzzy with a continuous boundary. First of all, it has been assumed that the size of the spatial memory in the fuzzy is known beforehand. In real-life engineering structures, the spatial memory is often unknown and relatively difficult to measure. Further, the method of determining the equivalent coupling factor only applies for fairly simple master structures, where its vibration pattern can be approximated as one or more sinusoidals in the midfrequency range. Also important is the estimation of the distribution of resonating mass per unit frequency. It is clear that practical methods for determining these fuzzy parameters are still needed.

${ }^{1}$ C. Soize, "Probabilistic structural modeling in linear dynamic analysis of complex mechanical systems, part I," (English edition), Rech. Aerosp. 5, 23-48 (1986).

${ }^{2}$ F. Chabas, A. Desanti, and C. Soize, "Probabilistic structural modelling in linear dynamic analysis of complex mechanical systems, part II," (English edition), Rech. Aerosp. 5, 49-67 (1986)

${ }^{3} \mathrm{C}$. Soize, "A model and numerical method in the medium frequency range for vibroacoustic predictions using the theory of structural fuzzy," J. Acoust. Soc. Am. 94, 849-865 (1993).

${ }^{4}$ J. Ormondroyd and J. P. Den Hartog, "Theory of dynamic vibration ab- sorber," Trans. ASME 50, APM-241 (1928).

${ }^{5}$ Noise and Vibration, edited by R. G. White and J. G. Walker (Ellis Horwood, Chichester, England, 1982), Chap. 25.

${ }^{6}$ D. F. Mead, Passive Vibration Control (Wiley, Chichester, England, 1999), Chap. 8.

${ }^{7}$ L. Friis and M. Ohlrich, "Vibration modelling of structural fuzzy with continuous boundary," J. Acoust. Soc. Am. 123, 718-728 (2008).

${ }^{8}$ A. D. Pierce, V. W. Sparrow, and D. A. Russell, "Fundamental structuralacoustic idealizations for structures with fuzzy internals," J. Vibr. Acoust. 117, 339-348 (1995)

${ }^{9}$ M. Strasberg and D. Feit, "Vibration damping of large structures induced by attached small resonant structures," J. Acoust. Soc. Am. 99, 335-344 (1996).

${ }^{10} \mathrm{G}$. Maidanik and K. J. Becker, "Various loss factors of a master harmonic oscillator coupled to a number of satellite harmonic oscillators," J. Acoust. Soc. Am. 103, 3184-3194 (1998).

${ }^{11}$ G. Maidanik and K. J. Becker, "Characterization of multiple-sprung masses for wideband noise control," J. Acoust. Soc. Am. 106, 3109-3118 (1999).

${ }^{12}$ G. Maidanik and K. J. Becker, "Criteria for designing multiple-sprung masses for wideband noise control," J. Acoust. Soc. Am. 106, 3119-3127 (1999).

${ }^{13}$ G. Maidanik and K. J. Becker, "Dependence of the induced loss factor on the coupling forms and coupling strengths: linear analysis," J. Sound Vib. 266, 15-32 (2003).

${ }^{14}$ G. Maidanik and K. J. Becker, "Induced noise control," J. Sound Vib. 277, 1041-1058 (2004).

${ }^{15}$ R. L. Weaver, "The effect of an undamped finite degree of freedom "fuzzy" substructure: Numerical solutions and theoretical discussion," J. Acoust. Soc. Am. 100, 3159-3164 (1996).

${ }^{16}$ A. Carcaterra and A. Akay, "Transient energy exchange between a primary structure and a set of oscillators: Return time and apparent damping," J. Acoust. Soc. Am. 115, 683-696 (2004).

${ }^{17}$ C. Soize, "Estimation of fuzzy substructure model parameters using the mean power flow equation of the fuzzy structure," J. Vibr. Acoust. 120, 279-286 (1998).

${ }^{18} \mathrm{C}$. Soize, "Estimation of fuzzy structure parameters for continuous junctions," J. Acoust. Soc. Am. 107, 2011-2020 (2000).

${ }^{19}$ A. D. Pierce, "Resonant-frequency-distribution of internal mass inferred from mechanical impedance matrices, with application to fuzzy structure theory," J. Vibr. Acoust. 119, 324-333 (1997).

${ }^{20}$ G. Maidanik, K. J. Becker, and L. J. Maga, "Replacement of a summation by an integration in structural acoustics," J. Sound Vib. 291, 323-348 (2006).

${ }^{21}$ L. Cremer, M. Heckl, and E. E. Ungar, Structure-Borne Sound (SpringerVerlag, Berlin, 1988).

${ }^{22}$ R. Cook, D. S. Malkus, M. F. Plesha, and R. J. Witt, Concepts and Applications of Finite Element Analysis (Wiley, New York, 2002).

${ }^{23}$ M. V. Drexel and J. H. Ginsberg, "Modal overlap and dissipation effects of a cantilever beam with multiple attached oscillators," J. Vibr. Acoust. 123, 181-187 (2001). 